\title{
Métodos físicos e cal hidratada para manejo do cascudinho dos aviários
}

\author{
Physical methods and hydrated lime for management of lesser mealworm
}

\author{
Jônatas Wolf ${ }^{\mathrm{I}}$ Alfredo de Gouvea ${ }^{\mathrm{II}}$ Everton Ricardi Lozano da Silva ${ }^{\mathrm{II}}$ \\ Michele Potrich $^{\mathrm{I}{ }^{*}}$ Alessandra Appel ${ }^{\mathrm{III}}$
}

RESUMO

A estabilidade de ambiência na avicultura moderna tem favorecido a proliferação de insetos praga. Nesse cenário, Alphitobius diaperinus (Panzer) (Coleoptera: Tenebrionidae), comumente denominado cascudinho, surge como principal inseto cosmopolita infestando camas de aviários. Neste sentido o objetivo deste trabalho foi avaliar os fatores cal hidratada, temperatura e umidade na cama de aviários sobre a mortalidade de A. diaperinus em condições de laboratório. Foram realizados bioensaios laboratoriais simulando o ambiente de criação em recipientes com a adição de cama de aviário, ração e adultos e larvas de A. diaperinus. A variável resposta foi a mortalidade aos sete e 10 dias após a instalação dos experimentos. A adição de cal hidratada na cama de aviário na dosagem de $400 \mathrm{~g} \mathrm{~m}^{-2}$ promoveu mortalidade parcial de adultos $(35,62 \% \pm 2,74)$ e larvas $(45,62 \% \pm 1,75)$ de $\boldsymbol{A}$. diaperinus. $O$ fator umidade na cama de aviário, independente do percentual, não provocou mortalidade significativa de adultos de A. diaperinus. A temperatura da cama de aviário em $45^{\circ} \mathrm{C}$ proveu mortalidade total de adultos e larvas de A. diaperinus nas condições propostas.

Palavras-chave: Alphitobius diaperinus, fatores abióticos, condicionador químico, avicultura industrial.

\section{ABSTRACT}

The stability of in the modern poultry industry has been favored the proliferation of insect pests. In this scenario, Alphitobius diaperinus (Panzer, 1797) (Coleoptera: Tenebrionidae), commonly known as lesser mealworm, emerges as major cosmopolitan insect infesting poultry litter. In this sense, the objective of this study was to evaluate the factors hydrated lime, temperature and humidity in the poultry litter on the A. diaperinus mortality under laboratory conditions. Laboratory bioassays were performed, simulating the poultry environment with litter in each experimental unit, with the addition of adults and larvae of A. diaperinus. The response variable was mortality at seven and 10 days after installation of the experiments. The addition of hydrated lime in the poultry litter at a dosage of $400 \mathrm{~g} \mathrm{~m}^{-2}$ promoted partial mortality of the adult population $(35.62 \% \pm 2,74)$ and larvae $(45.62 \% \pm 1,75)$ of $\boldsymbol{A}$. diaperinus. The moisture in the litter, regardless of percentage, did not cause significant mortality of adults of A. diaperinus. The temperature in the litter at $45^{\circ} \mathrm{C}$ caused total mortality of adults and larvae of A. diaperinus at the conditions studied.

Key words: Alphitobius diaperinus, abiotic factors, chemical conditioner, poultry industry.

\section{INTRODUÇÃO}

O cascudinho de aviários, Alphitobius diaperinus (Panzer) (Coleoptera: Tenebrionidae), é um inseto considerado praga cosmopolita de importância particular como vetor e reservatório de diversos agentes patogênicos e parasitas de aves (DUNFORD \& KAUFMAN, 2009), de maneira especial, bactérias dos gêneros Salmonella, Campylobacter e Clostridium (VITTORI et al., 2007; HAZELEGER et al., 2008; ROCHE et al., 2009; LEFFER et al., 2010). Desta feita, afetam a saúde das aves e a consequente qualidade da produção de alimentos.

O sistema criatório vigente tem favorecido a proliferação de $\boldsymbol{A}$. diaperinus em aviários de aves de corte. Isso se deve, principalmente, pela constância

'Programa de Pós-graduação em Zootecnia, Produção Animal, Universidade Tecnológica Federal do Paraná (UTFPR), Dois Vizinhos, PR, Brasil.

"ICoordenação de Ciências Biológicas, UTFPR, Estrada para Boa Esperança, Km 4, CP 157, 85660-000, Dois Vizinhos, PR, Brasil. E-mail: michelepotrich@utfpr.edu.br.*Autor para correspondência.

II'Brasil Foods (BRF), Dois Vizinhos, PR, Brasil. 
da faixa de temperatura de operação no interior dos galpões, pelos níveis de umidade naturalmente observados na cama, pelo reaproveitamento desta no intervalo de lotes e pela disponibilidade de alimento e refúgio para os insetos (UEMURA et al., 2008).

A temperatura ambiental e a umidade relativa da cama desempenham papel na regulação da oviposição e eclosão de ovos de A. diaperinus, sobremaneira interferindo na plasticidade ecológica do comportamento reprodutivo dessa espécie, afetando assim sua população adulta. A temperatura no interior da cama e dos galpões de criação de frangos, com variação entre $18^{\circ} \mathrm{C}$ e $32^{\circ} \mathrm{C}$, objetivando atender zona de conforto térmico para as aves, está sempre em um patamar que possibilita o ótimo desenvolvimento de A. diaperinus. Ademais, a média de temperatura na cama, $25,3^{\circ} \mathrm{C}$ a $30,7^{\circ} \mathrm{C}$, associada ao acúmulo de matéria-prima, propicia multiplicação de microorganismos saprofíticos (SARIN \& SAXENA, 1973; UEMURA et al., 2008).

A utilização de condicionadores químicos na cama de aviário, como a cal hidratada, a cal virgem, o sulfato de alumínio e o gesso agrícola, entre outros, revelam-se alternativas para o controle de $\boldsymbol{A}$. diaperinus. Estas têm efeitos relacionados a mudanças nos gradientes de umidade na cama, produção de gases no interior das instalações, controle de micro-organismos de interesse epidemiológico e alteração das propriedades do adubo residual da atividade (WATSON et al., 2003; DAI PRA et al., 2009; WOLF et al., 2012). A cal hidratada, especificamente, tem sido utilizada como dessecante, absorvendo o excesso de umidade e promovendo a secagem, criando assim um ambiente hostil para insetos e patógenos (WATSON et al., 2003).

Diante do exposto, há necessidade de desenvolver programas de manejo com a integração de métodos de controle para reduzir populações de $\boldsymbol{A}$. diaperinus nos sistemas de produção avícola. Nesse sentido, o objetivo deste trabalho foi avaliar o impacto da cal hidratada, umidade e temperatura sobre a mortalidade de adultos e larvas de A. diaperinus na cama de aviário.

\section{MATERIAL E MÉTODOS}

Os insetos foram obtidos de aviários infestados em cama sem tratamento químico e a cama obtida de aviários comerciais com "camas de meia idade" (quatro lotes), sendo o substrato maravalha de pinus. A cama original foi analisada para determinação das características físico-químicas de $\mathrm{pH}$, umidade, matéria mineral, matéria orgânica e concentração de amônia $\left(\mathrm{NH}_{3}\right)$.
Para a realização dos bioensaios, cada caixa plástica (Unidade Experimental - UE), provida de tampa com encaixe $(12 \mathrm{~L}, 30 \mathrm{~cm}$ de comprimento $\times 20 \mathrm{~cm}$ de largura $\times 20 \mathrm{~cm}$ de altura), foi abastecida com $0,003 \mathrm{~m}^{3}$ de cama de aviário, totalizando 0,6 $\mathrm{kg}$ de material, valor correspondente à camada de cama utilizada na cobertura de pisos de aviários comerciais ( $5 \mathrm{~cm}$ de espessura). Cada UE recebeu $10 \mathrm{~g}$ de ração comercial de frangos de corte, atendendo às especificações nutricionais. Foram adicionadas 20 larvas do 3 o ao 7o ínstar (entre sete e 10mm) e 20 adultos (WOLF et al., 2012a) em cada UE. Realizaram-se três experimentos sequenciais e independentes, avaliando os fatores cal hidratada, umidade e temperatura na cama de aviário.

No primeiro experimento, avaliou-se o impacto da adição de cal hidratada, sendo que cada UE recebeu, de maneira casualizada, a cal distribuída sobre a cama, nas dosagens correspondentes de $0,200,400$ e $600 \mathrm{~g} \mathrm{~m}^{-2}$. As dosagens de cal foram definidas em equivalência às proporções utilizadas na superfície do substrato de camas em aviários comerciais.

No segundo experimento, o parâmetro umidade foi analisado por meio da introdução de água destilada na proporção do peso do substrato nas UE, sendo que os tratamentos constaram de quatro umidades $(25 \%, 45 \%, 65 \%$ e $85 \%)$. No tratamento testemunha, manteve-se o percentual original da cama in natura, acrescendo umidade proporcionalmente nos demais tratamentos.

No terceiro experimento, a variável temperatura foi avaliada como fator causal de mortalidade de A. diaperinus. Foram avaliadas as temperaturas de $15^{\circ} \mathrm{C}, 30^{\circ} \mathrm{C}, 45^{\circ} \mathrm{C}$ e $60^{\circ} \mathrm{C}$. A definição das temperaturas foi baseada para 15 e $30^{\circ} \mathrm{C}$, amplitude térmica utilizada em aviários comerciais para conforto das aves e, para 45 e $60^{\circ} \mathrm{C}$, de acordo com limites extremos obtidos com a fermentação da cama de aviários nos intervalos entre lotes. Para tal, a cama contendo A. diaperinus foi acondicionada em saco plástico transparente autoclavável e submetida a $24 \mathrm{~h}$ de exposição térmica, conforme tratamentos e então devolvida às UE. As UE foram acondicionadas em sala climatizada à temperatura de $21 \pm 5^{\circ} \mathrm{C}$ e umidade relativa $65 \pm 5 \%$. As temperaturas máxima e mínima foram registradas com termohigrômetro digital no ambiente controlado, também foram mensurados $\mathrm{pH}$ e temperatura nos tempos zero, um, sete e 10 dias.

O delineamento experimental foi inteiramente casualizado, esquema fatorial $4 \times 2$, sendo quatro valores de cada variável estudada [cal 
hidratada (três concentrações + controle), umidade e temperatura] e dois tempos de avaliação (sete e dez dias), com quatro repetições. A variável resposta foi a mortalidade de larvas e adultos após sete e dez dias da instalação dos experimentos. A avaliação constou da quantificação dos insetos mediante triagem das UE, identificando larvas e adultos, coletados com pinça e colocados em placas de Petri identificadas. Os dados de insetos vivos remanescentes na UE foram convertidos por diferença em percentual de mortos sobre a amostra inicial (20 adultos e 20 larvas) e transformados em ARCSEN $\sqrt{ }(\mathrm{x} / 100)$. Estes foram submetidos à análise de variância (teste F) e as médias comparadas pelo teste de Tukey, 5\% de significância (ASSISTAT, Versão 7.6 beta, SILVA \& AZEVEDO, 2011).

\section{RESULTADOS E DISCUSSÃO}

Avaliação do efeito da adição de cal na cama de aviário sobre $A$. diaperinus

No que cinge a interação entre as dosagens de cal e os diferentes tempos de quantificação de mortalidade para adultos, não foi aplicado o teste de comparação de médias, pois o $\mathrm{F}$ de interação não foi significativo ( F 0,6805 P>0,050). A análise isolada dos fatores demonstrou diferença na mortalidade de adultos entre os tempos sete e 10 dias, com 22,50 e $28,13 \%$ (F 8,4539 P 0,0131), respectivamente, e também diferenças quanto ao fator concentrações de cal hidratada (F 16,2165 $\mathrm{P}<0,001)$. Observou-se percentual médio de mortalidade crescente para adultos. Não obstante, os tratamentos 400 e $600 \mathrm{~g} \mathrm{~m}^{-2}$, com $35,62 \%$ e $40,62 \%$ de mortalidade, respectivamente, diferiram significativamente da testemunha $(11,25 \%)$, sem, no entanto, diferirem entre si (Tabela 1). De acordo com WATSON et al. (2003), em bioensaio similar ao presente estudo, a adição de $600 \mathrm{~g} \mathrm{~m}^{-2}$ de cal hidratada proporcionou mortalidade de $26,6 \%$ e $66,5 \%$ de adultos e larvas de A. diaperinus.

Para as larvas, a interação foi significativa (F 4,2017 P 0,0301), acenando para a elevação da mortalidade conforme elevação concomitante da dosagem de cal hidratada e tempo de quantificação pós-aplicação. Porém, como observado para adultos, não houve diferença significativa na mortalidade entre os tratamentos 400 e $600 \mathrm{~g} \mathrm{~m}^{-2}$, indicando que a utilização da cal para controlar $A$. diaperinus pode ser realizada na dosagem de $400 \mathrm{~g}$ $\mathrm{m}^{-2}$, já que a mortalidade na dosagem de $200 \mathrm{~g} \mathrm{~m}^{-2}$ não diferiu da testemunha para larvas e adultos (Tabela 1).

Dedução possível para o resultado de redução observada diz respeito à mudança do gradiente iônico do substrato. Isso pode ser observado nas aferições de concentração de íons de hidrogênio $(\mathrm{pH})$ efetuadas no tempo, na qual se obteve, no dia seguinte à aplicação, valores de 8,$51 ; 9,14 ; 10,06$ e 10,85 , respectivamente, para as dosagens 0, 200, 400 e 600g m $\mathrm{g}^{-2}$. Segundo WATSON et al. (2003), a sustentação do $\mathrm{pH}$ em valores $\geq 11$ por mais de 96 horas pode ser responsável pelo efeito de controle de A. diaperinus. De acordo com os autores, essas implicações decorrem de distúrbios da permeabilidade e tonicidade nos insetos, bem como no equilíbrio hidroeletrolítico. O presente trabalho obteve resultados de controle do inseto com alteração do gradiente acima de 10, sustentando o fato de que $\mathrm{pH}$, já acima destes patamares, pode exercer alterações no equilíbrio hidroeletrolítico do inseto.

Ainda, colige-se como fator causal da mortalidade o efeito higroscópico da cal. Neste atinente, a cal absorve a umidade periférica do inseto e resseca sua estrutura, dificultando a locomoção e alimentação. Assim, o modo de ação se dá pela

Tabela 1 - Porcentagem média de mortalidade ( \pm EP) de adultos e larvas de Alphitobius diaperinus em cama de aviários, com diferentes dosagens de cal hidratada, acumulados aos sete e 10 dias. - DV. Temperatura $21 \pm 5^{\circ} \mathrm{C}$ e Umidade Relativa $65 \pm 5 \%$.

\begin{tabular}{|c|c|c|c|}
\hline \multirow{2}{*}{ Tratamentos } & \multirow{2}{*}{ Mortalidade adultos (\%) } & \multicolumn{2}{|c|}{-Mortalidade larvas (\%)- } \\
\hline & & 7 dias & 10 dias \\
\hline Testemunha & $11,3 \pm 1,3 b$ & $18,8 \pm 3,2 \mathrm{cA}$ & $20,0 \pm 3,5 \mathrm{bA}$ \\
\hline $200 \mathrm{~g} \mathrm{~m}^{-2}$ & $13,8 \pm 1,5 b$ & $31,3 \pm 3,8 \mathrm{bcA}$ & $31,3 \pm 3,8 \mathrm{bA}$ \\
\hline $400 \mathrm{~g} \mathrm{~m}^{-2}$ & $35,6 \pm 2,7 \mathrm{a}$ & $43,8 \pm 3,2 \mathrm{abA}$ & $47,5 \pm 1,4 \mathrm{aB}$ \\
\hline $600 \mathrm{~g} \mathrm{~m}^{-2}$ & $40,6 \pm 5,1 \mathrm{a}$ & $51,3 \pm 3,2 \mathrm{aA}$ & $58,8 \pm 3,8 \mathrm{aB}$ \\
\hline$P$ & $<0,001$ & $<0,0301$ & \\
\hline $\mathrm{CV}(\%)$ & 11,7 & 2,50 & \\
\hline
\end{tabular}

Médias seguidas pela mesma letra minúscula na coluna e letra maiúscula na linha não diferem significativamente entre si pelo teste de Tukey $(\mathrm{P}<0,05)$. Dados originais - para análise estatística foram transformados em $\operatorname{ARCSEN} \sqrt{ }(\mathrm{x} / 100)$. 
desidratação ou dessecação, uma vez que partículas do pó aderem ao corpo dos insetos e ocorre a remoção da cera epicuticular, devido à abrasão no tegumento ou a adsorção. Esse efeito também pode ser obtido, segundo DAI PRA et al. (2009), porque a cal promove redução da atividade de água $\left(\mathrm{a}_{\mathrm{w}}\right)$ na cama, reduzindo assim a água livre.

Avaliação do efeito de diferentes umidades na cama de aviário sobre $A$. diaperinus

Observou-se que os percentuais de umidade avaliados na cama de aviário não causaram mortalidade significativa de adultos de $A$. diaperinus, comparando-se à testemunha (F 0,7237 $\mathrm{P}>0,050)$, bem como os dados de quantificação entre os tempos (F 3,2555 P 0,0962) (Tabela 2). Para a fase larval, verificou-se que o tratamento com $45 \%$ de umidade causou mortalidade de $41,87 \%$, valor superior aos demais tratamentos (Tabela 2). Independente das implicações da umidade no ciclo biológico do inseto na fase larval, não se observou diferença quanto ao fator tempo sete e 10 dias (F 4,0654 P 0,0666). VICTOR \& OGONOR (1987) observaram que os adultos preferem condições secas no ambiente de criação, sendo a umidade de $20 \%$ favorável ao desenvolvimento de adultos de A. diaperinus. Já AGABOU \& ALLOUI (2012) encontraram preferência de A. diaperinus para umidades entre 30$40 \%$ no substrato da cama.

A resposta para variações na umidade da cama é influenciada por alterações comportamentais diferenciadas na ecologia de adultos e larvas. Esse efeito é referendado por SALIN et al. (2000), os quais identificaram que a distribuição espacial de adultos de $\boldsymbol{A}$. diaperinus está relacionada à alta umidade do solo, baixa compactação e densidade da superfície e, excetuando-se a umidade do solo, o mesmo ocorre com larvas e pupas. Esse fato denota a interferência do percentual de umidade no ciclo biológico de $\boldsymbol{A}$. diaperinus, como demonstrado por vários estudos (WILSON \& MINER, 1969; SALIN et al., 2000; UEMURA et al., 2008).

No presente trabalho, a manipulação da umidade, enquanto fator isolado, não promoveu $\mathrm{o}$ controle de A. diaperinus. O uso da umidade em camas de aviários de aves de corte prevê acelerar o processo fermentativo da cama quando esta é amontoada no interior do aviário. Além disso, é importante salientar que os efeitos derivados desta prática impactam em outros fatores importantes, como volatilização de amônia, alteração do $\mathrm{pH}$ e elevação temporária da temperatura (DAI PRA \& ROLL, 2012).

Avaliação do efeito de diferentes temperaturas na cama de aviário sobre $A$. diaperinus

A interação entre as diferentes temperaturas e tempos sete e 10 dias apresentou $\mathrm{F}$ significativo para adultos (F 3,8493 P 0,0373) e para larvas ( F 7,6505 P 0,004) (Tabela 3). As médias de mortalidade para o fator temperatura na testemunha $\left(15^{\circ} \mathrm{C}\right)$ e $30^{\circ} \mathrm{C}$ não diferiram entre si, tanto para adultos, quanto para larvas (Tabela 3). Dessa forma, conjectura-se que temperaturas nesse limiar não causam mortalidade significativa de $\boldsymbol{A}$. diaperinus. Esses dados são semelhantes aos resultados obtidos por WILSON \& MINER (1969), nos quais a temperatura de $32^{\circ} \mathrm{C}$ foi determinada como ótima para o desenvolvimento de ovos até adultos de A. diaperinus. CHERNAKI \& ALMEIDA (2001) obtiveram sobrevivência de 66,7\% e $86,7 \%$ para larvas na temperatura experimental de $22^{\circ} \mathrm{C}$ e $31^{\circ} \mathrm{C}$, respectivamente. Ainda, de acordo com os autores, temperaturas inferiores a $16,5^{\circ} \mathrm{C}$ interrompem o ciclo de $A$. diaperinus em seus diferentes estágios, já que, nesta faixa de temperatura, não ocorre desenvolvimento de imaturos e a quantidade de insetos é menor.

A mortalidade de A. diaperinus foi total, independente do tempo de quantificação, a partir

Tabela 2 - Porcentagem média de mortalidade $( \pm \mathrm{EP})$ de adultos e larvas de Alphitobius diaperinus em cama de aviários, com diferentes umidades. Laboratório de Controle Biológico - UTFPR - DV. Temperatura $21 \pm 5^{\circ} \mathrm{C}$ e Umidade Relativa $65 \pm 5 \%$.

\begin{tabular}{lcr}
\hline Tratamentos & Mortalidade adultos (\%) & Mortalidade larvas (\%) \\
\hline $25 \%$ & $2,5 \pm 0,9 \mathrm{a}$ & $35,6 \pm 6,1 \mathrm{~b}$ \\
$45 \%$ & $8,8 \pm 2,8 \mathrm{a}$ & $41,9 \pm 9,6 \mathrm{a}$ \\
$65 \%$ & $5,6 \pm 2,0 \mathrm{a}$ & $28,8 \pm 3,2 \mathrm{c}$ \\
$85 \%$ & $6,3 \pm 2,3 \mathrm{a}$ & $26,9 \pm 4,6 \mathrm{c}$ \\
$\mathrm{P}$ & $>0,05$ & $>0,05$ \\
$\mathrm{CV}(\%)$ & 4,78 & 29,88 \\
\hline
\end{tabular}

Médias seguidas pela mesma letra minúscula, na coluna, não diferem significativamente entre si pelo teste de Tukey (P<0,05). Dados originais - para análise estatística foram transformados em ARCSEN $\sqrt{ }$ (x/100). 
Tabela 3 - Porcentagem média de mortalidade $( \pm \mathrm{EP})$ de adultos e larvas de Alphitobius diaperinus em cama de aviários, com diferentes temperaturas, acumulados aos sete e 10 dias. Laboratório de Controle Biológico $-\mathrm{UTFPR}-\mathrm{DV}$. Temperatura $21 \pm 5^{\circ} \mathrm{C}$ e Umidade Relativa $65 \pm 5 \%$.

\begin{tabular}{|c|c|c|c|c|}
\hline \multirow{2}{*}{ Tratamentos } & \multicolumn{4}{|c|}{----Mortalidade adultos (\%)---------------- } \\
\hline & 7 dias & 10 dias & 7 dias & 10 dias \\
\hline $15^{\circ} \mathrm{C}$ & $5 \pm 2,9 \mathrm{bA}$ & $15 \pm 2 \mathrm{bB}$ & $33,8 \pm 5,2 \mathrm{bA}$ & $47,5 \pm 3,2 \mathrm{bB}$ \\
\hline $30^{\circ} \mathrm{C}$ & $13,8 \pm 6,3 \mathrm{bA}$ & $16,3 \pm 6,3 \mathrm{bA}$ & $31,3 \pm 12,5 \mathrm{bA}$ & $41,3 \pm 14,6 \mathrm{bB}$ \\
\hline $45^{\circ} \mathrm{C}$ & $100 \mathrm{aA}$ & $100 \mathrm{aA}$ & $100 \mathrm{aA}$ & $100 \mathrm{aA}$ \\
\hline $60^{\circ} \mathrm{C}$ & $100 \mathrm{aA}$ & $100 \mathrm{aA}$ & $100 \mathrm{aA}$ & $100 \mathrm{aA}$ \\
\hline $\mathrm{P}$ & $<0,0373$ & & & $<0,004$ \\
\hline CV $(\%)$ & 10,67 & & & 47,90 \\
\hline
\end{tabular}

Médias seguidas pela mesma letra minúscula na coluna e letra maiúscula na linha não diferem significativamente entre si pelo teste de Tukey $(\mathrm{P}<0,05)$. Dados originais - para análise estatística foram transformados em ARCSEN $\sqrt{ }(\mathrm{x} / 100)$.

de $45^{\circ} \mathrm{C}$, para larvas e adultos. A possibilidade de utilizar a temperatura como barreira para prevenir a migração da praga foi investigada por SCHMITZ \& WOHLGEMUTH (1988), os quais observaram que adultos e larvas raramente persistem no ambiente quando as temperaturas excedem os $45^{\circ} \mathrm{C}$, fato corroborado em laboratório por GAZONI et al. (2012). De acordo com VORHEES \& BRADLEY (2012), os limites térmicos afetam a abundância e a distribuição de insetos; a temperatura crítica máxima é superior em adultos do que em larvas e pupas, bem como a mortalidade que ocorre em temperaturas superiores em adultos.

A utilização de métodos físicos é estratégia para o manejo de cascudinhos. Nesse sentido, VORIS et al. (1994) determinam que práticas aplicadas na cama de aviário, com a variação da temperatura, afetam a dinâmica populacional de A. diaperinus. FIORENTIN (2005) afirma que a fermentação da cama, mediante amontoamento, atinge, na maioria das vezes, temperatura de $60^{\circ} \mathrm{C}$, configurando-se como alternativa viável para obtenção de temperaturas elevadas na prática avícola. Segundo DAI PRA \& ROLL (2012), o enlonamento reduz enterobactérias e controla vetores, como A. diaperinus, já que a cama amontoada e coberta pode propiciar temperatura superior a $60^{\circ} \mathrm{C}$. Assim sendo, a associação de métodos físicos deve ser investigada para manejo e controle do cascudinho dos aviários.

\section{CONCLUSÃO}

A adição de cal hidratada na cama de aviário, na dosagem de $400 \mathrm{~g} \mathrm{~m}^{-2}$, reduz o percentual de adultos e larvas de $\boldsymbol{A}$. diaperinus. A temperatura de $45^{\circ} \mathrm{C}$ na cama de aviário provê mortalidade totalitária de adultos e larvas de A. diaperinus nas condições avaliadas.

\section{REFERÊNCIAS}

AGABOU, A.; ALLOUI, N. Spatio-temporal variability of broiler house litter infestation with Alphitobius diaperinus during three successive breeding cycles in the North-East of Algeria. International Journal of Current Research, v.4, n.8, p.140-142, 2012.

CHERNAKI-LEFFER, A.M.; ALMEIDA, L.M. Exigências térmicas, período de desenvolvimento e sobrevivência de imaturos de Alphitobius diaperinus (Panzer) (Coleoptera: Tenebrionidae). Neotropical Entomology, v.30, n.3, p.365-368, 2001. Disponível em: $<$ http://www.scielo.br/scielo.php?pid=S1519566X2001000300004\&script=sci_arttext $>$. Acesso em: 21 fev. 2013. doi:10.1590/S1519-566X2001000300004.

DAI PRA, M.A. et al. Uso de cal virgem para o controle de Salmonella spp. e Clostridium spp. em camas de Aviário. Ciência Rural, v.39, n.4, p.1189-1194, 2009. Disponível em: <http://www. scielo.br/scielo.php?script=sci_arttex\&pid=S01038478200900040 0035\&Ing=en\&nrm=iso $>$. Acesso em: 15 jul. 2012. doi: $10.1590 /$ S0103-84782009005000028.

DAI PRA, M.A.; ROLL, V.F.B. (Orgs.). Cama de aviário: utilização, reutilização e destino. Porto Alegre: Manas/Evangraf, 2012. $86 \mathrm{p}$.

DUNFORD, J.C.; KAUFMAN, P.E. Lesser mealworm, litter beetle, Alphitobius diaperinus (Panzer) (Insecta: Coleoptera: Tenebrionidae). Entomology and Nematology Department, Florida Cooperative Extension Service, Institute of Food and Agricultural Sciences, University of Florida. Jun. 2009. Acesso em: 22 fev. 2013. Online. Disponível em: <http://edis.ufl.edu/ pdffiles/IN/IN66200.pdf>.

FIORENTIN, L. Reutilização da cama de frangos e as implicações de ordem bacteriológica na saúde humana e animal. Concórdia: Embrapa Suínos e Aves, 2005. 23p. (Documentos, 94).

GAZONI, F.L. et al. Avaliação da resistência do cascudinho (Alphitobius diaperinus) (Panzer) (Coleoptera: Tenebrionidae) a diferentes temperaturas. Arquivos do Instituto Biológico, v.79, n.1, p.69-74, 2012.

HAZELEGER, W.C. et al. Darkling beetles (Alphitobius diaperinus) and their larvae as potential vectors for the transfer of 
Campylobacter jejuni and Salmonella enterica serovar paratyphi B variant java between successive broiler flocks. Applied and Environmental Microbiology, v.74, p.6887-6891, 2008 Disponível em: <http://aem.asm.org/cgi/reprint/74/22/6887>. Acesso em: 02 jan. 2013. doi: 10.1128/AEM.00451-08.

LEFFER, A.M. et al. Vectorial competence of larvae and adults of Alphitobius diaperinus in the transmission of Salmonella enteritidis in poultry. Vector-Borne and Zoonotic Diseases, v.10 p.481-487, 2010. Disponível em: <http://www.ncbi.nlm.nih.gov/ pubmed/19929223>. Acesso em: 02 jan. 2013. doi: 10.1089 vbz.2008.0089.

ROCHE, A.J. et al. Transmission of Salmonella to broilers by contaminated larval and adult lesser mealworms, Alphitobius diaperinus (Coleoptera: Tenebrionidae). Poultry Science, v.88, n.1, p.44-48, 2009. Disponível em: <http:/www.ncbinlm.nih. gov/pubmed/19096055>. Acesso em: 22 fev. 2013. doi: 10.3382/ ps.2008-00235

SALIN, C. et al. Spatial distribution of Alphitobius diaperinus (Panzer) (Coleoptera: Tenebrionidae) in the soil of a poultry house along a breeding cycle. European Journal of Soil Biology, v.36, n.2, p.107-115, 2000. Disponível em: <http://www.sciencedirect. com/science/article/pii/S1164556300010542>. Acesso em: $20 \mathrm{dez}$. 2012. doi: 10.1016/S1164-5563(00)01054-2.

SARIN, K.; SAXENA, S.C. Effect of temperature and relative humidity on the development and mortality of Alphitobius diaperinus (Panz), a stored product pest. Folia Biologica (Krakow), v.21, p.223-228, 1973

SCHMITZ, V.M.; WOHLGEMUTH, R. Investigations on mass increase and behavior of Alphitobius diaperinus Panz. (Coleoptera,Tenebrionidae) in poultry houses as a basis for directed control in practice. Anzeiger fuer Schaedlingskunde und Pflanzenschutz, v.61, p.108-114, 1988.

SILVA, F. de A.S.; AZEVEDO, C.A.V. de. ASSISTAT Versão 7.6 beta (2011). Disponível em: Homepage <http://www.assistat. com.> DEAG-CTRN-UFCG - Atualiz. 14 maio 2012.

UEMURA, D.H. et al. Distribuição e dinâmica populacional do cascudinho Alphitobius diaperinus (Coleoptera: Tenebrionidae) em aviários de frango de corte. Arquivos do Instituto Biológico, v. 75 , n.4, p.429-435, 2008.
VICTOR, J.R.; OGONOR, P.S. Humidity reactions in the two species of tenebrionid beetles infesting poultry and food storage houses in Nigeria. Revue de Zoologie Africaine, v.101, p.423430, 1987.

VITTORI, J. et al. Alphitobius diaperinus como veiculador de Clostridium perfringens em granjas avícolas do interior paulista. Ciência Rural, v.37, n.3, p.894-896, 2007. Disponível em: < http:// www.scielo.br/scielo.php?script $=$ sci arttext\&pid $=$ S0 1038478200 7000300048\&lng=en\&nrm=iso $>$. Acesso em: 21 fev. 2013. doi: 10.1590/S0103-84782007000300048.

VORHEES, A.S.; BRADLEY, T.J. Differences in critical thermal maxima and mortality across life stages of the mealworm beetle Tenebrio molitor. Journal of Experimental Biology, v.215, p.2319-2326, 2012. Disponível em: <http://jeb.biologists.org/content/215/13/2319.long>. Acesso em: 24 fev. 2013. doi: 10.1242/jeb.070342.

VORIS, J.C. et al. Temperature affects lesser mealworm populations in turkey brooder houses. California Agriculture, v.48, p.18-21, 1994. Disponível em: <http://ucanr.org/repository/cao/landingpage. cfm?article=ca.v048n02p18\&fulltext=yes $>$. Acesso em: 24 fev. 2013. doi: 10.3733/ca.v048n02p18.

WATSON, D.W. et al. Effects of lime hydrate on the growth and development of darkling beetle, Alphitobius diaperinus. International Journal of Poultry Science, v.2, p.91-96, 2003. Disponível em: $<\mathrm{http}$ //scialert.net/abstract/?doi=ijps.2003.91.96>. Acesso em: $23 \mathrm{fev}$. 2013. doi: 10.3923/ijps.2003.91.96.

WILSON, T.H.; MINER, F.D. Influence of temperature on development of the lesser mealworm, Alphitobius diaperinus (Coleoptera: Tenebrionidae). Journal Kansas Entomology Society., v.42, p.294303, 1969.

WOLF, J. et al. Utilização de cal hidratada na cama de aviário para o controle de Alphitobius diaperinus (Coleoptera: Tenebrionidae), em condições de laboratório. In: CONGRESSO BRASILEIRO DE ENTOMOLOGIA, 12, 2012, Curitiba, PR. Anais... Curitiba: CBE, 2012. p.24.

WOLF, J. et al. Proposta de metodologia para avaliação de bioensaios para o controle de Alphitobius diaperinus em condições de laboratório. In: CONGRESSO BRASILEIRO DE ENTOMOLOGIA, 12., 2012, Curitiba, PR. Anais... Curitiba: CBE, 2012a. p.21. 